\title{
Levels of selected pro- and anti-inflammatory cytokines in cerebrospinal fluid in patients with hydrocephalus
}

\author{
Kinga Czubowicz ${ }^{1^{*}}$, Mariusz Głowacki ${ }^{*}$, Ewa Fersten ${ }^{3,4}$, Ewa Kozłowska ${ }^{5}$, Robert P. Strosznajder ${ }^{1}$, \\ Zbigniew Czernicki' \\ ${ }^{1}$ Laboratory of Preclinical Research and Environmental Agents, Department of Neurosurgery, Mossakowski Medical Research \\ Centre, Polish Academy of Sciences, Warsaw, ${ }^{2}$ Department of Neurosurgery, John Paul II Western Hospital, Grodzisk Mazowiecki, \\ ${ }^{3}$ Department of Neurosurgery, Maria Sklodowska-Curie Memorial Oncology Center, Poland, ${ }^{4}$ Institute of Applied Psychology, \\ The Maria Grzegorzewska Academy of Special Education, ${ }^{5}$ Department of Immunology, Faculty of Biology, University of Warsaw, \\ Warsaw, ${ }^{6}$ Department of Neurosurgery, Second Faculty of Medicine, Medical University of Warsaw, Warsaw, Poland \\ *Authors equally contributed to this study.
}

\begin{abstract}
Cytokines are widely known mediators of inflammation accompanying many neurodegenerative disorders including normal pressure hydrocephalus (NPH). NPH is caused by impaired cerebrospinal fluid (CSF) absorption and treated by surgical shunt insertion. The early diagnosis of NPH is difficult because of various manifestations of the disease. One of the most promising research directions is biochemical CSF analysis. The aim of this study was to determine the CSF levels of cytokines. The levels of various cytokines (IL-6, IL-8, IL-12, IL-10 and TNF- $\alpha$ ) were measured in patients with idiopathic active normal pressure hydrocephalus, arrested hydrocephalus and hydrocephalus with brain atrophy compared to controls. Our study showed that the concentrations of IL-6 and IL-8 were significantly elevated in the group with idiopathic active hydrocephalus compared to control patients. Moreover, we observed that the levels of IL-6 and IL-8 in the group with idiopathic active hydrocephalus were significantly higher compared to patients with arrested hydrocephalus and hydrocephalus with brain atrophy.
\end{abstract}

Key words: hydrocephalus, cerebrospinal fluid, cytokines, inflammation.

\section{Introduction}

Hydrocephalus comprises a heterogeneous group of conditions which have in common almost only dilatation of cerebral spinal fluid spaces. The etiological diagnosis of hydrocephalus is essential for the prognosis. In a significant number of cases the cause cannot be identified. The following etiological factors are observed: congenital and acquired, i.e. infection, hemorrhage, tumor, increased venous sinus pressure, iatrogenic, idiopathic. Excluding obstructive non-communicating hydrocephalus in the adult population there are different terms describing abnormal accumulation of cerebrospinal fluid (CSF), i.e. normal 
pressure hydrocephalus, arrested hydrocephalus, and hydrocephalus ex vacuo (cerebral atrophy). Normal pressure hydrocephalus (NPH) is a disturbance of physiological balance between secretion and resorption of the CSF which can lead to its abnormal accumulation in the brain ventricular system with a normal range of CSF pressure. The result of this pathophysiological process is an enlargement of the ventricular system and serious dysfunction of the central nervous system. This syndrome was first described by Hakim and Adams [12]. The classic symptoms comprising Hakim's triad for NPH are cognitive decline, gait disturbances and urinary incontinence. Symptomatology of NPH is not always complete and unequivocal. It is very diverse and often limited to only one symptom. Therefore it is hard to distinguish it from other central nervous system disorders. Arrested and ex vacuo hydrocephalus have different symptomatology and dynamics of symptoms. NPH can mimic or occur in combination with various elderly age-related disorders such as cerebrovascular disease, neurodegenerative diseases (e.g. Alzheimer's, Parkinson's), urological disorders, spinal stenosis and others (e.g. arrested hydrocephalus) $[10,33,37]$. Proper diagnosis has an extremely important impact on further diagnostic and treatment procedures $[3,7,9]$. The treatment of choice in symptomatic NPH is surgical. The surgical treatment is intended to drain CSF from the cerebral ventricles using different CSF shunt systems. Despite the great development of radiological imaging methods and many other diagnostic methods and functional tests, hydrocephalus remains a diagnostic and therapeutic challenge. There are no widely accepted criteria for NPH diagnosis. Some authors have suggested that the only reliable diagnosis is to document a positive response to shunt placement, but other studies showed a poor correlation between shunt responsiveness and the selection criteria now used $[13,16]$. NPH is routinely diagnosed on the basis of convergent evidence from clinical history, examination, psychological tests and brain imaging, and the lumbar infusion test with determination of CSF outflow resistance. Additional tests may help to improve diagnostic confidence, differential diagnosis and prognosis of shunt responsiveness. One of the most promising research directions is biochemical CSF analysis. CSF participates in the free exchange of many biochemical products with the brain and provides an insights into changes in the brain milieu associated directly with the patient's condition $[30,41,44]$.
Cytokines are a diverse group of polypeptides comprising interleukins (IL), interferons (INF) tumor necrosis factors (TNF), chemokines and growth factors. Cytokine levels are rapidly up-regulated in response to various diseases, injuries and infections $[2,35,40]$. Cytokines have typically been classified as either pro-inflammatory or anti-inflammatory. In relation to the neurodegenerative processes the major pro-inflammatory cytokines are known to be IL-1 $\beta$, IL-6, IL-8 and TNF- $\alpha$. The main anti-inflammatory cytokines are IL-4 and IL-10 [40,41].

The aim of this study was to determine the CSF levels of the cytokines IL-6, IL-8, IL-12, IL-10 and TNF- $\alpha$ in patients with idiopathic active normal pressure hydrocephalus, arrested hydrocephalus and hydrocephalus with brain atrophy.

\section{Material and methods \\ Subjects}

Results of the clinical examination led to selection of patients with the following criteria (inclusion criteria): all patients presented enlargement (dilatation) of the brain ventricular system, with CSF pressure in the normal range, there were no additional diagnosed CNS disorders, and patient's mental status allowed complete neuropsychological tests to be performed (TMT Trail Making Test, Verbal Fluency Test - Controlled Oral Word Association Test, Wisconsin Card Sorting Test). All patients were examined according to the same protocol: neurological examination with gait analysis, neuropsychological tests, medical history of the diseases, brain imaging, lumbar infusion test.

The authors declare that in accordance with Polish law and regulations in Bielanski Hospital, Warsaw, Poland, all patients participating in this study signed individual informed consent before the lumbar procedure. In each case, the procedure was performed with strict medical indications and all samples were sent for diagnostic and scientific reasons.

Among this population the following 3 subgroups of patients were distinguished.

\section{Group I - active idiopathic normal pressure hydrocephalus (active iNPH)}

Five patients reported with progressive gait disturbances and cognitive deficits - mainly memory disorders. Those patients were admitted to hospital and qualified for brain imaging studies (CT, MR) 
because of motor deficits and headaches. Brain imaging showed ventricular system enlargement in all patients. In neurological examination gait disturbances were present. Neuropsychological examinations (tests) showed specific cognitive deficits, mainly serious disorders of visuospatial and visuoperceptual abilities in visual discrimination and visual counting tasks, which suggests impairment in visual form perception or constructive function. There were also memory deficits, especially word recognition and word recall. According to the knowledge and experience of the authors as well as the literature, those deficits were typical for iNPH $[8,13,14,28]$. All patients were diagnosed with the lumbar infusion test introduced by Katzman [15] and achieved results in the range $12-14 \mathrm{mmHg} / \mathrm{ml} / \mathrm{min}$, which indicated a significant CSF outflow resistance and suggested a benefit from shunting. Finally all patients were diagnosed with idiopathic active hydrocephalus and were qualified for shunt placement.

\section{Group II - arrested hydrocephalus (AH)}

Six patients with ventricular system enlargement were observed for several years. In some cases information from the patient history suggested that this process started in early childhood or even was congenital. Patients in this group were generally in a good status, without cognitive deficits and only with mild neurological disorders. In the neuropsychological tests patients did not present cognitive deficits. In some cases with a low level of intellectual functioning since early childhood there were only single symptoms of inertia of nervous processes, and worsening of verbal fluency and operational memory span. Those symptoms were not characteristic for iNPH. The lumbar infusion test resulted in outflow resistance in the range $6-8 \mathrm{mmHg} / \mathrm{ml} / \mathrm{min}$ (with the exception of 1 case in which outflow resistance was higher, up to $10 \mathrm{mmHg} / \mathrm{ml} / \mathrm{min}$ ), which confirmed that there were no abnormalities in CSF resorption. Patients in this group were not qualified for shunting and were diagnosed as having "arrested" hydrocephalus (AH).

\section{Group III - hydrocephalus with brain atrophy}

Five patients had ventricular system dilatation in the course of brain atrophy (BA), where vacant spaces were passively filled with CSF. Neuropsychological tests showed impairment of affective-motivational regulation, disorders involving reduced psychomotor drive, slowness and emotional liability, cognitive deficits, mostly in the form of concentration deficiency, impaired shifting in motor sequences (dynamic praxis disturbance), limited verbal fluency and a short and visuospatial memory deficits. Reductions in operational memory span and learning ability, mainly verbal, were also sporadically seen.

\section{Control group}

The control group consisted of two patients, who because of other clinical indications were subjected to lumbar puncture and a sample of CSF was collected. A part of the CSF sample was used in this study. The first patient was admitted to the hospital with suspected rhinorrhea resulting from a minor head trauma four weeks earlier. No CNS pathology was found during the brain imaging (either immediately after the injury or on admission a month later). The neurological examination did not reveal pathology. The results of all other tests were in the normal range; therefore rhinorrhea or another CNS pathology was excluded. The second patient was admitted with a history of severe headache and suspected idiopathic subarachnoid hemorrhage (SAH). Both CT scan and CSF analysis excluded SAH or another CNS pathology. It is worth underlining that in the control group inflammatory processes were not observed.

\section{Sample analysis}

For the evaluation of selected cytokine level (pg/ $\mathrm{ml}$ ) in the cerebrospinal fluid, the BD CBA Human Inflammatory Cytokines Kit was used. The kit is designated for quantitative and simultaneous measurement of the protein levels of interleukin (IL)- 6, IL-8, IL-10, IL-12 and tumor necrosis factor alpha (TNF- $\alpha$ ). The measurement was prepared following the manufacturer's instructions. Data were collected using a FACSCalibur (BD, San Jose, Ca, USA) flow cytometer and analyzed using FACS Compt Cytometric Bead Array software II (BD, San Jose, Ca, USA).

\section{Statistical analysis}

Analyses among multi-group data were conducted using one-way analysis of variance (ANOVA), followed by the Newman-Keuls post hoc test. Data are given as the means \pm SEM. $P$ values $<0.05$ were considered statistically significant. The number of values $(n)$ was between 4 and 12 . 
Table I. The levels of cytokines in control group, active iNPH, arrested hydrocephalus and hydrocephalus with brain atrophy

\begin{tabular}{|lcccc|}
\hline Cytokines $(\mathrm{pg} / \mathrm{ml})$ & Control & $\begin{array}{c}\text { Active idiopathic } \\
\text { hydrocephalus }\end{array}$ & $\begin{array}{c}\text { Arrested hydrocephalus } \\
\text { IL-10 }\end{array}$ & $\begin{array}{c}\text { Hydrocephalus with } \\
\text { brain atrophy }\end{array}$ \\
\hline $\mathrm{IL}-12$ & $1.32 \pm 0.063$ & $1.37 \pm 0.084$ & $1.38 \pm 0.080$ & $1.34 \pm 0.068$ \\
\hline TNF- $\alpha$ & $1.37 \pm 0.120$ & $1.66 \pm 0.121$ & $1.55 \pm 0.096$ & $1.44 \pm 0.068$ \\
\hline Data represent mean values + SEM & $1.50 \pm 0.108$ & $1.40 \pm 0.113$ & $1.47 \pm 0.059$ & $1.36 \pm 0.085$ \\
\hline
\end{tabular}

\section{Results}

In this study we investigated the concentration of pro-inflammatory cytokines in idiopathic active normal pressure hydrocephalus, arrested hydrocephalus and hydrocephalus with brain atrophy. The levels of analyzed CSF biomarkers (IL-10 and IL-12, TNF- $\alpha$ ) were similar and did not differ significantly between the control and the three groups of patients.

We observed that the levels of IL-10 and TNF- $\alpha$ concentrations were around $1.4-1.5 \mathrm{pg} / \mathrm{ml}$ in all three groups. Also, the mean IL-12 concentration was in the range $1.4-1.6 \mathrm{pg} / \mathrm{ml}$ in the group of hydro-

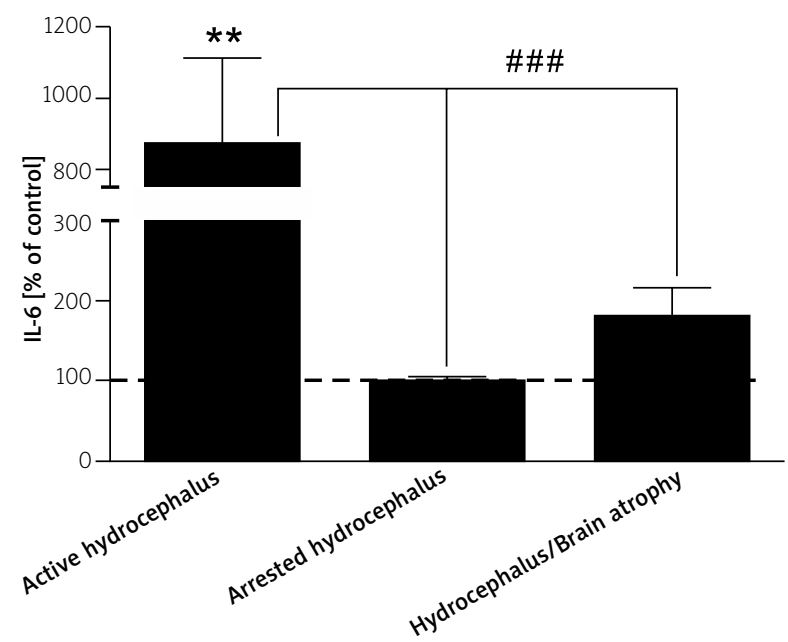

Fig. 1. Level of IL-6 in active idiopathic normal pressure hydrocephalus (iNPH) (active hydrocephalus), arrested hydrocephalus and in hydrocephalus with brain atrophy. Data represent mean values \pm S.E.M. ${ }^{* *} p<0.01$ versus control group (marked with a dashed line) by one-way ANOVA followed by the Newman-Keuls test. $\# \#$ \# 0.001 versus active iNPH by one-way ANOVA followed by the Newman-Keuls test. cephalus with brain atrophy, active iNPH and arrested hydrocephalus in order of increasing values. The levels of these cytokines are reported in Table I.

Interestingly, our study showed that the concentrations of IL- 6 and IL- 8 were significantly elevated in the group with idiopathic active hydrocephalus compared to control patients (Figs. 1 and 2). The values of IL-6 and IL-8 in the control group in our study correspond to those observed in other studies [24,25,29]. Moreover, the mean concentrations of IL- 6 and IL- 8 in the group with active iNPH were significantly higher than in patients with arrested hydrocephalus and hydrocephalus with brain atrophy.

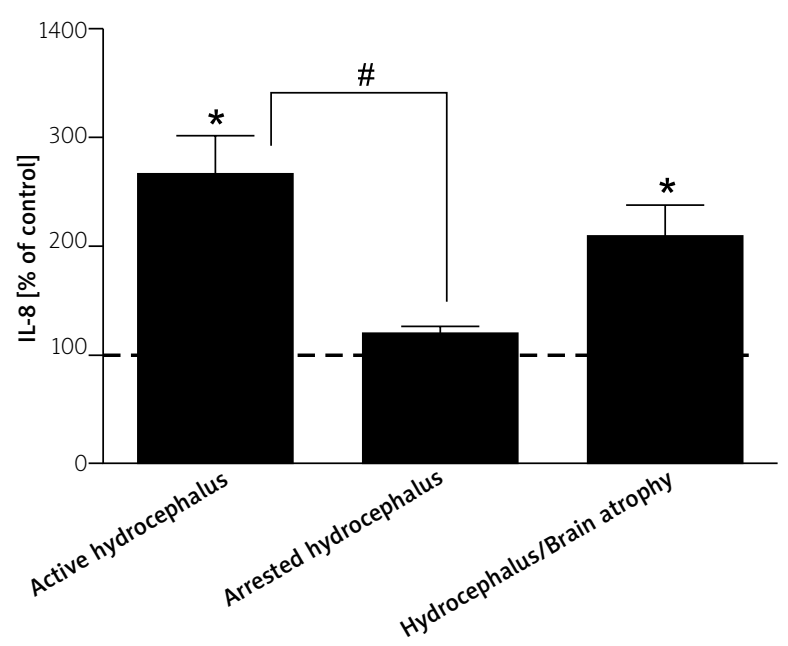

Fig. 2. Level of IL-8 in active idiopathic normal pressure hydrocephalus (iNPH) (active hydrocephalus), arrested hydrocephalus and in hydrocephalus with brain atrophy. Data represent mean values \pm S.E.M. ${ }^{*} p<0.05$ versus control group (marked with a dashed line) by one-way ANOVA followed by the Newman-Keuls test. ${ }^{\#} p<0.05$ versus active iNPH by one-way ANOVA followed by the Newman-Keuls test. 


\section{Discussion}

Systemic and brain inflammatory processes are associated with various neurodegenerative and neuropsychiatric diseases, for example Alzheimer's disease, Parkinson's disease, multiple sclerosis, schizophrenia and normal pressure hydrocephalus (NPH) $[6,28,36,38]$. Inflammation usually contributes to disease progression through the production of pro-inflammatory mediators. Cytokines are the best known mediators of inflammation and play important roles within and outside the brain. Different levels of cytokines in CSF were reported in various types of adult hydrocephalus [17,19,20,32,34,42,43]. In our study we distinguished three different groups of NPH patients: with active idiopathic hydrocephalus, arrested hydrocephalus and hydrocephalus with brain atrophy. It was interesting whether those different types of hydrocephalus should be associated with various concentrations of cytokines in CSF.

In our group of subjects with active iNPH, the CSF level of IL-6 was significantly elevated compared to control. This is consistent with studies reported by other authors $[17,42]$.

Sosvorova [42] observed that the CSF level of IL- 6 was significantly increased on the first day of lumbar drainage in NPH patients compared to controls. After that the level decreased and approached the control value. Low levels of IL-6 are observed in the brain under physiological conditions. IL-6 plays a pivotal role in the normal homeostasis of neuronal tissue, and its absence leads to reduced glial activation in traumatic brain injury $[18,31]$. On the other hand, a rapid increase in expression and secretion of IL-6 is observed in various neurological diseases, for example Alzheimer's and Parkinson's disease [5], brain cancer [21], multiple sclerosis [11,23] and brain ischemia [1]. In our study the level of IL-6 was significantly lower in patients with arrested and with brain atrophy hydrocephalus compared to the group with active iNPH.

The brain production of IL- 6 is up-regulated by TNF- $\alpha$ [45]. The level of TNF- $\alpha$ in CSF is increased in multiple sclerosis $[38,39,40]$ and Parkinson's disease $[26,27]$. Controversial data have been reported concerning NPH subjects $[19,20,32,42,43]$. In our study the CSF levels of TNF- $\alpha$ did not differ between the control group and the three patient groups.

Chemokines play an essential role in neuroinflammation as mediators of leukocyte infiltration.
IL-8 is a pro-inflammatory chemokine and its overexpression has been observed in various neurological disorders, such as multiple sclerosis and Alzheimer's disease $[4,22]$. In our study we also observed significant changes in the CSF level of IL-8 in the group of active iNPH compared to the control group. Moreover, the concentration of IL-8 was significantly lower in patients with arrested hydrocephalus compared to the group with active iNPH. Killer [17] observed an increase in IL-8 level in patients developing hydrocephalus following hemorrhage. In the study performed by Pyykko [32] the lumbar CSF samples showed higher IL-8 levels compared to ventricular samples in NPH patients.

In our study no differences were observed between the control group and the three patient groups in the level of another pro-inflammatory cytokine, IL-12. Also other authors have not observed any changes in $\mathrm{NPH}$ patients compared to controls [32,34].

The CSF level of IL-10, an anti-inflammatory cytokine, which is able to suppress the production of IL- $1 \beta$ and TNF- $\alpha$, did not change in our three groups of patients, which is in line with other results [32,34]. The study by Sosvorova [42] showed an increase in IL-10 CSF levels on the first day of lumbar drainage in NPH patients compared to control groups, but on the third day the level decreased.

In conclusion, the significantly elevated levels of cytokines IL-6 and IL-8 in CSF suggest inflammatory changes in the brain and could be taken into consideration as biomarkers for idiopathic active normal pressure hydrocephalus.

\section{Acknowledgments}

This study was supported by the statutory budget of the Mossakowski Medical Research Centre, Polish Academy of Sciences (Theme No. 1).

\section{Disclosure}

Authors report no conflict of interest.

\section{References}

1. Ali C, Nicole O, Docagne F, Lesne S, MacKenzie ET, Nouvelot A, Buisson A, Vivien D. Ischemia-induced interleukin- 6 as a potential endogenous neuroprotective cytokine against NMDA receptor-mediated excitotoxicity in the brain. J Cereb Blood Flow Metab 2000; 20: 956-966.

2. Allan SM, Rothwell NJ. Cytokines and acute neurodegeneration. Nat Rev Neurosci 2001; 2: 734-744. 
3. Aoki Y, Kazui H, Tanaka T, Ishii R, Wada T, Ikeda S, Hata M, Canuet L, Katsimichas T, Musha T, Matsuzaki H, Imajo K, Kanemoto H, Yoshida T, Nomura K, Yoshiyama K, Iwase M, Takeda M. Noninvasive prediction of shunt operation outcome in idiopathic normal pressure hydrocephalus. Sci Rep 2015; 5: 7775.

4. Bajetto A, Bonavia R, Barbero S, Florio T, Schettini G. Chemokines and their receptors in the central nervous system. Front Neuroendocrinol 2001; 22: 147-184.

5. Benveniste EN. Cytokine actions in the central nervous system. Cytokine Growth Factor Rev 1998; 9: 259-275.

6. Blum-Degen D, Müller T, Kuhn W, Gerlach M, Przuntek H, Riederer $P$. Interleukin-1 beta and interleukin- 6 are elevated in the cerebrospinal fluid of Alzheimer's and de novo Parkinson's disease patients. Neurosci Lett 1995; 202: 17-20.

7. Brean A, Eide PK. Assessment of idiopathic normal pressure patients in neurological practice: the role of lumbar infusion testing for referral of patients to neurosurgery. Eur J Neurol 2008; 15: 605-612.

8. Czepko R. Wielokierunkowa ocena wodogłowia komunikującego oraz model postępowania. Wydawnictwo Uniwersytetu Jagielońskiego, Kraków 2000 [In Polish].

9. Czosnyka Z, Czosnyka M, Owler B, Momjian S, Kasprowicz M, Schmidt EA, Smielewski P, Pickard JD. Clinical testing of CSF circulation in hydrocephalus. Acta Neurochir Suppl 2005; 95: 247-251.

10. Czosnyka Z, Owler B, Keong N, Santarius T, Baledent O, Pickard JD, Czosnyka M. Impact of duration of symptoms on CSF dynamics in idiopathic normal pressure hydrocephalus. Acta Neurol Scand 2011; 123: 414-418.

11. Frei K, Fredrikson S, Fontana A, Link H. Interleukin-6 is elevated in plasma in multiple sclerosis. J Neuroimmunol 1991; 31: 147-153.

12. Hakim S, Adams RD. The special clinical problem of symptomatic hydrocephalus with normal cerebrospinal fluid pressure. Observations on cerebrospinal fluid hydrodynamics. J Neurol Sci 1965; 2: 307-327.

13. Hebb AO, Cusimano MD. Idiopathic normal pressure hydrocephalus: a systematic review of diagnosis and outcome. Neu rosurgery 2001; 49: 1166-1184.

14. Jeppsson A, Zetterberg H, Blennow K, Wikkels $\varnothing$ C. Idiopathic normal-pressure hydrocephalus: pathophysiology and diagnosis by CSF biomarkers. Neurology 2013; 80: 1385-1392.

15. Katzman R, Hussey F. A simple constant-infusion manometric test for measurement of CSF absorption. I. Rationale and method. Neurology 1970; 20: 534-544.

16. Kiefer M, Unterberg A. The differential diagnosis and treatment of normal-pressure hydrocephalus. Dtsch Arztebl Int 2012; 109 15-25.

17. Killer M, Arthur A, Al-Schameri AR, Barr J, Elbert D, Ladurner G, Shum J, Cruise G. Cytokine and growth factor concentration in cerebrospinal fluid from patients with hydrocephalus following endovascular embolization of unruptured aneurysms in comparison with other types of hydrocephalus. Neurochem Res 2010; 35: 1652-1658.

18. Klein MA, Möller JC, Jones LL, Bluethmann H, Kreutzberg GW, Raivich G. Impaired neuroglial activation in interleukin-6 deficient mice. Glia 1997; 19: 227-233.

19. Lee JH, Park DH, Back DB, Lee JY, Lee Cl, Park KJ, Kang SH, Cho TH, Chung YG. Comparison of cerebrospinal fluid biomark- ers between idiopathic normal pressure hydrocephalus and subarachnoid hemorrhage-induced chronic hydrocephalus: a pilot study. Med Sci Monit 2012; 18: PR19-25.

20. Leinonen V, Menon LG, Carroll RS, Dello lacono D, Grevet J, Jääskeläinen JE, Black PM. Cerebrospinal fluid biomarkers in idiopathic normal pressure hydrocephalus. Int J Alzheimers Dis 2011; 2011: 312526.

21. Lilja A, Nordborg C, Brun A, Salford LG, Aman P. Expression of the IL-6 family cytokines in human brain tumors. Int J Oncol 2001; 19: 495-499.

22. Liu C, Cui G, Zhu M, Kang X, Guo H. Neuroinflammation in Alzheimer's disease: chemokines produced by astrocytes and chemokine receptors. Int J Clin Exp Pathol 2014; 7: 8342-8355.

23. Maimone D, Guazzi GC, Annunziata P. IL-6 detection in multiple sclerosis brain. J Neurol Sci 1997; 146: 59-65.

24. Matejčíková Z, Mareš J, Sládková V, Svrčinová T, Vysloužilová J, Zapletalová J, Kaňovský P. Cerebrospinal fluid and serum levels of interleukin-8 in patients with multiple sclerosis and its correlation with O-albumin. Mult Scler Relat Disord 2017; 14: 12-15.

25. Mehta VK, Verma R, Garg RK, Malhotra HS, Sharma PK, Jain A. Study of interleukin- 6 and interleukin- 8 levels in patients with neurological manifestations of dengue. J Postgrad Med 2017; 63: 11-15.

26. Mogi M, Harada M, Kondo T, Riederer P, Inagaki H, Minami M, Nagatsu T. Interleukin-1 beta, interleukin-6, epidermal growth factor and transforming growth factor-alpha are elevated in the brain from parkinsonian patients. Neurosci Lett 1994; 180: 147-150.

27. Mogi $M$, Harada $M$, Narabayashi $H$, Inagaki $H$, Minami $M$, Nagatsu T. Interleukin (IL)-1 beta, IL-2, IL-4, IL-6 and transforming growth factor-alpha levels are elevated in ventricular cerebrospinal fluid in juvenile parkinsonism and Parkinson's disease. Neurosci Lett 1996; 211: 13-16.

28. Nassar BR, Lippa CF. Idiopathic Normal Pressure Hydrocephalus: A Review for General Practitioners. Gerontol Geriatr Med 2016; 2: 2333721416643702

29. Oda Y, Tsuruta R, Kasaoka S, Inoue T, Maekawa T. The cutoff values of intrathecal interleukin 8 and 6 for predicting the neurological outcome in cardiac arrest victims. Resuscitation 2009; 80: 189-193.

30. Palmqvist S, Zetterberg H, Mattsson N, Johansson P; Alzheimer's Disease Neuroimaging Initiative, Minthon L, Blennow K, Olsson M, Hansson O; Swedish BioFINDER Study Group. Detailed comparison of amyloid PET and CSF biomarkers for identifying early Alzheimer disease. Neurology 2015; 85: 1240-1249.

31. Penkowa M, Moos T, Carrasco J, Hadberg H, Molinero A, Bluethmann $H$, Hidalgo J. Strongly compromised inflammatory response to brain injury in interleukin-6-deficient mice. Glia 1999; 25: 343-357.

32. Pyykkö OT, Lumela M, Rummukainen J, Nerg O, Seppälä TT, Herukka SK, Koivisto AM, Alafuzoff I, Puli L, Savolainen S, Soininen $\mathrm{H}$, Jääskeläinen JE, Hiltunen $M$, Zetterberg $H$, Leinonen V. Cerebrospinal fluid biomarker and brain biopsy findings in idiopathic normal pressure hydrocephalus. PLoS One 2014; 9: e91974. 
33. Relkin N, Marmarou A, Klinge P, Bergsneider M, Black PM. Diagnosing idiopathic normal-pressure hydrocephalus. Neurosurgery 2005; 57: S4-16.

34. Rota E, Bellone G, Rocca P, Bergamasco B, Emanuelli G, Ferrero P. Increased intrathecal TGF-beta1, but not IL-12, IFN-gamma and IL-10 levels in Alzheimer's disease patients. Neurol Sci 2006; 27: 33-39.

35. Rothwell NJ, Strijbos PJ. Cytokines in neurodegeneration and repair. Int J Dev Neurosci 1995; 13: 179-185.

36. Sasayama D, Hattori K, Wakabayashi C, Teraishi T, Hori H, Ota M, Yoshida S, Arima K, Higuchi T, Amano N, Kunugi H. Increased cerebrospinal fluid interleukin-6 levels in patients with schizophrenia and those with major depressive disorder. J Psychiatr Res 2013; 47: 401-406.

37. Savolainen S, Karhu J, Pääkkönen A, Paljärvi L, Partanen J, Alafuzoff I, Vapalahti M. Auditory event-related potentials differentiate patients with normal pressure hydrocephalus and patients with concomitant Alzheimer's disease verified by brain biopsy. Neuroreport 2001; 12: 33-37.

38. Seppi D, Puthenparampil M, Federle L, Ruggero S, Toffanin E, Rinaldi F, Perini P, Gallo P. Cerebrospinal fluid IL-1 $\beta$ correlates with cortical pathology load in multiple sclerosis at clinical onset. J Neuroimmunol 2014; 270: 56-60

39. Sharief MK, Hentges R. Association between tumor necrosis factor-alpha and disease progression in patients with multiple sclerosis. N Engl J Med 1991; 325: 467-472.

40. Smith JA, Das A, Ray SK, Banik NL. Role of pro-inflammatory cytokines released from microglia in neurodegenerative diseases. Brain Res Bull 2012; 87: 10-20.

41. Sosvorova L, Mohapl M, Vcelak J, Hill M, Vitku J, Hampl R. The impact of selected cytokines in the follow-up of normal pressure hydrocephalus. Physiol Res 2015; 64: S283-290.

42. Sosvorova L, Vcelak J, Mohapl M, Vitku J, Bicikova M, Hampl R. Selected pro- and anti-inflammatory cytokines in cerebrospinal fluid in normal pressure hydrocephalus. Neuro Endocrinol Lett 2014; 35: 586-593.

43. Tarkowski E, Tullberg M, Fredman P, Wikkelsö C. Normal pressure hydrocephalus triggers intrathecal production of TNFalpha. Neurobiol Aging 2003; 24: 707-714.

44. Tarnaris A, Watkins LD, Kitchen ND. Biomarkers in chronic adult hydrocephalus. Cerebrospinal Fluid Res 2006; 3: 11.

45. Tobinick E, Gross H, Weinberger A, Cohen H. TNF-alpha modulation for treatment of Alzheimer's disease: a 6-month pilot study. MedGenMed 2006; 8: 25. 